\title{
ALGEBRAIC $K$-THEORY AND DERIVED EQUIVALENCES SUGGESTED BY T-DUALITY FOR TORUS ORIENTIFOLDS
}

\author{
JONATHAN ROSENBERG \\ In honor of the birthday of my good friend Chuck Weibel
}

\begin{abstract}
We show that certain isomorphisms of (twisted) $K R$-groups that underlie T-dualities of torus orientifold string theories have purely algebraic analogues in terms of algebraic $K$-theory of real varieties and equivalences of derived categories of (twisted) coherent sheaves. The most interesting conclusion is a kind of Mukai duality in which the "dual abelian variety" to a smooth projective genus- 1 curve over $\mathbb{R}$ with no real points is (mildly) noncommutative.
\end{abstract}

\section{INTRODUCTION}

1.1. Background. This paper grew out of joint work with Charles Doran and Stefan Mendez-Diez [12, 13], in which we studied type II orientifold string theories on circles and 2-tori. In these theories, D-brane charges lie in twisted $K R$-groups of $(X, \iota)$, where $X$ is the spacetime manifold and $\iota$ is the involution on $X$ defining the orientifold structure. (That D-brane charges for orientifolds are classified by $K R$-theory was pointed out in [47, §5.2], [19], and [18], but twisting (as defined in $[26,25,27]$ and [12]) may arise due to the $B$-field, as in [48], and/or the charges of the $O$-planes, as explained in [12].) These orientifold theories were found in [13] to split up into a number of T-duality groupings, with the theories in each grouping all related to one another by various T-dualities. The twisted $K R$-groups attached to each of the theories within a T-duality grouping were all found to be isomorphic to one another, up to a degree shift. Actual isomorphisms of the twisted $K R$-groups for the theories within a T-duality group were constructed in [40], where we showed that all of these $K$-theory isomorphisms arise from cases of the real Baum-Connes conjecture for solvable (in fact, also virtually abelian) discrete groups. (By the way, these are cases where the real Baum-Connes conjecture was already known to hold, by $[5,41]$.) This sort of duality involving $K R$-theory already appeared

2010 Mathematics Subject Classification. Primary 14F05; Secondary 19E08 19L64 81T30 $14 \mathrm{~F} 22$ 14H60 14H81.

Key words and phrases. orientifold, KR-theory, elliptic curve, twisting, Mukai duality, Tduality, derived category, coherent sheaves, algebraic $K$-theory, real algebraic geometry, Azumaya algebra.

Partially supported by NSF grant DMS-1206159. 
in [38, Theorem 2.5] in the case where the group involved is free abelian. In that theorem, real Baum-Connes was shown to give an isomorphism from $K O$-homology of a torus to $K R$-cohomology of another torus of the same dimension (really the T-dual torus). This isomorphism turns out to be related to T-duality between the type I and type IA string theories on the circle.

The work of Karoubi and Weibel [22] showed that $K R$-theory of complex algebraic varieties (equipped with an antiholomorphic involution) is closely related to the algebraic $K$-theory of the associated real algebraic variety. This raised the tantalizing possibility that T-duality of orientifolds is closely related to isomorphisms of algebraic $K$-groups of real varieties or to equivalences of derived categories of (twisted) coherent sheaves on real varieties. The purpose of the paper is to verify this conjecture in the case of real affine rational curves (modeling orientifolds on the circle) and real elliptic curves.

After the preliminary Section 1.3, we begin in Section 2 with the case of certain rational affine curves over $\mathbb{R}$, which give algebraic models for the four orientifolds on the circle. We compute the algebraic $K$-theory for all of these "algebraic circles." While the $K$-theory spectra for dual algebraic circles are not exactly the same, they do become equivalent after introducing finite coefficients. Then in Section 3, we consider an algebraic analogue of the duality between the type IIA orientifold theory on a Klein bottle and the $\widetilde{\mathrm{IA}}$ theory, which lives on a split elliptic curve over $\mathbb{R}$ with a nontrivial twist given by a certain Azumaya algebra, described in Proposition 4. The main result, Theorem 5, is an equivalence of derived categories of twisted coherent sheaves, which is considerably stronger (see for example [45, Example V.3.10.2]) than a mere isomorphism of algebraic $K$-groups. This result can be interpreted as saying that the "dual abelian variety" to a smooth projective genus- 1 curve over $\mathbb{R}$ with no real points is a noncommutative Azumaya algebra over a split elliptic curve over $\mathbb{R}$. This result is in many ways reminiscent of the result of Căldăraru [7, Chapter 4], [8] that in the case of an elliptic fibration without a section, a certain Azumaya algebra over the relative Jacobian is dual to the original elliptic fibration, and there is an associated equivalence of derived categories of [twisted] coherent sheaves. (The genus-1 curve with no real points is like an elliptic fibration without a section, and the split elliptic curve is its Jacobian, as we will see in Section 3.) The result is also closely related to the results of [3], though it doesn't quite seem to be covered by the hypotheses imposed there. After this paper was completed, we learned that Theorem 5 , in a different formulation, has also been proved in [23] using very different methods.

1.2. Notational convention. Since this paper uses both topological and algebraic $K$-theory, we need a way to distinguish them. $K$-groups with the index up, such as $K^{-\bullet}, K O^{-\bullet}, K R^{-\bullet}$, and $K S C^{-\bullet}$, always denote topological $K$-groups. $K$-groups with the index down, such as $K_{\bullet}(R)$, always denote algebraic $K$-theory of rings or schemes. (All the schemes discussed here will be smooth, so it will 
not be necessary to distinguish this from $G$-theory or $K^{\prime}$-theory.) When we need topological $K$-homology (this only shows up once in the paper), we denote it by $K_{\bullet}^{\text {top }}$. The notation $\mathbb{K}$ denotes the algebraic $K$-theory spectrum (of a ring, scheme, etc.), and $\mathbb{K}^{\text {top }}$ denotes the (non-connective) topological $K$-theory spectrum.

1.3. Review of KR-theory. We quickly remind the reader about Atiyah's $K R$ theory [4]. This is the topological $K$-theory (with compact supports) of Real vector bundles $E$ over Real spaces $(X, \iota)$. A Real space is just a locally compact Hausdorff space $X$ equipped with a self-homeomorphism $\iota$ satisfying $\iota^{2}=\mathrm{id}_{X}$. A Real vector bundle $E$ over such a space is a complex vector bundle equipped with a conjugate-linear vector bundle automorphism of period 2 compatible with $\iota$. The $K R$-theory of $(X, \iota)$ can be identified with the topological $K$-theory of the real Banach algebra $C_{0}(X, \iota)=\left\{f \in C_{0}(X) \mid f(\iota(x))=\overline{f(x)}\right\}$. We shall use the indexing convention of $[24,12,13]: \mathbb{R}^{p, q}$ denotes $\mathbb{R}^{p} \oplus \mathbb{R}^{q}$ with the involution that is +1 on the first summand and -1 on the second summand, and $S^{p, q}$ denotes the unit sphere in $\mathbb{R}^{p, q}$. Thus $S^{0,2}$, for example, denotes the circle $S^{1}$ with its antipodal involution. The two indices $p$ and $q$ play complementary roles, in that $K R^{\bullet}\left(X \times \mathbb{R}^{p, q}\right) \cong K R^{\bullet-p+q}(X)[4$, Theorem 2.3]. Atiyah [4, Proposition 3.5] also showed that for any locally compact space $X, K R^{-\bullet}\left(X \times S^{0,2}\right) \cong K S C^{-\bullet}(X)$, the self-conjugate $K$-theory of Anderson [2] and Green [16].

Karoubi and Weibel [22, Main Theorem 4.8] showed that $K R$-theory is intimately connected with algebraic $K$-theory of algebraic varieties defined over $\mathbb{R}$. In fact, if $X$ is a smooth projective variety defined over $\mathbb{R}$, then $K_{\bullet}(X, \mathbb{Z} / m) \cong$ $K R^{-\bullet}(X(\mathbb{C}), \mathbb{Z} / m)$ in the stable range $\bullet \geq \operatorname{dim} X$. Here the set $X(\mathbb{C})$ of complex points is given its (compact Hausdorff) analytic topology and the natural action of $G=\operatorname{Gal}(\mathbb{C} / \mathbb{R})$. They proved the theorem for $m$ a power of two, which is the most interesting case, though it also follows for $m$ an odd prime by Bloch-Kato. The theorem holds for affine varieties as well, provided one modifies the definition of $K R^{-\bullet}(X(\mathbb{C}))$ a bit. Since $X(\mathbb{C})$ is noncompact in this case, one needs to use homotopy-theoretic $K R$-theory $K R_{h}$ instead of $K R$-theory with compact supports. $\left(K R_{h}^{-\bullet}(X) \cong K O^{-\bullet}\right.$ (pt) if $X$ is equivariantly, but not necessarily properly, contractible, so in this theory, all affine spaces $\mathbb{A}^{n}(\mathbb{R})$ behave like $\operatorname{Spec} \mathbb{R}$.)

\section{Algebraic Circle orientifolds}

2.1. An algebraic analogue of circle orientifolds. We begin with the case of orientifolds on a circle, with the involution coming from a linear involution on $\mathbb{R}^{2}$, restricted to the unit circle. It was found in $[14,12,13]$ that there are four such orientifold theories, known in the physics literature as types I, $\widetilde{\mathrm{I}}, \mathrm{IA}$, and $\widetilde{\mathrm{IA}}$. These split into two T-duality groupings, one of which contains theories I and IA, corresponding to the Real spaces $S^{2,0}$ and $S^{1,1}$, and the other of which contains theories $\widetilde{\mathrm{I}}$ and $\widetilde{\mathrm{IA}}$, corresponding to the Real spaces $S^{0,2}$ and $S_{(+,-)}^{1,1}$. Here the subscript $(+,-)$ in $S_{(+,-)}^{1,1}$ indicates that of the two $O$-planes in $S^{1,1}$ (i.e., fixed 
points for the involution), one has been given a plus sign (meaning that the ChanPaton bundle there is of real type) and one has been given a minus sign (meaning that the Chan-Paton bundle there is of quaternionic type).

What are the algebraic analogues of these Real spaces?

Let $W, X$, and $Y$ be the following smooth affine curves defined over $\mathbb{R}$ :

$$
\begin{aligned}
W & =\operatorname{Spec}\left(\mathbb{R}[x, y] /\left(x^{2}+y^{2}-1\right)\right), \\
X & =\operatorname{Spec}\left(\mathbb{R}[x, y] /\left(x^{2}+y^{2}+1\right)\right), \\
Y & =\operatorname{Spec}\left(\mathbb{R}\left[v, v^{-1}\right]\right) \cong \mathbb{G}_{m} .
\end{aligned}
$$

After applying base change $\square \rightsquigarrow$ Spec $\mathbb{C} \times_{\text {Spec } \mathbb{R}} \square, W, X$, and $Y$ all become $\operatorname{Spec}\left(\mathbb{C}\left[v, v^{-1}\right]\right) \cong \mathbb{G}_{m}$. For $Y$ this is obvious. For $X_{\mathbb{C}}$ we use the isomorphism sending $(x, y) \in \mathbb{A}^{2}(\mathbb{C})$ with $x^{2}+y^{2}+1=0$ to $x+i y \in \mathbb{G}_{m}(\mathbb{C})$; note that $z=x+i y$ is invertible and determines the pair $(x, y)$ since $(x+i y)(i y-x)=1$, hence $x=\left(z-z^{-1}\right) / 2$ and $y=\left(z+z^{-1}\right) /(2 i)$. Similarly, for $W_{\mathbb{C}}$ we use the isomorphism sending $(x, y) \in \mathbb{A}^{2}(\mathbb{C})$ with $x^{2}+y^{2}-1=0$ to $x+i y \in \mathbb{G}_{m}(\mathbb{C})$; note that $z=x+i y$ is invertible and determines the pair $(x, y)$ since $(x+i y)(x-i y)=1$, hence $x=\left(z+z^{-1}\right) / 2$ and $y=\left(z-z^{-1}\right) /(2 i)$.

In accordance with the point of view of Karoubi and Weibel, we view $W(\mathbb{C})$, $X(\mathbb{C})$ and $Y(\mathbb{C})$ as Real spaces in the sense of Atiyah. Of course in all cases we just have $\mathbb{C}^{\times}$as the underlying space, but the Galois action of $G=\operatorname{Gal}(\mathbb{C} / \mathbb{R})$ is different. On $Y(\mathbb{C})$, the action of $G$ is usual complex conjugation on $\mathbb{C}^{\times}$, and the fixed set (the union of the "O-planes") is $\mathbb{R}^{\times}=(-\infty, 0) \sqcup(0, \infty)$. Thus $Y$ is an algebraic model for the Real space $S^{1,1}$. On $X(\mathbb{C})$, on the other hand, the Galois action must be free, since $X(\mathbb{R})=\emptyset$. (There are no points $(x, y) \in \mathbb{A}^{2}(\mathbb{R})$ with $x^{2}+y^{2}+1=0$.) In fact one can see that when we identify $X(\mathbb{C})$ with $\mathbb{C}^{\times}, G$ acts by $z \mapsto-\bar{z}^{-1}$. $X(\mathbb{C})$ has a (non-proper) $G$-equivariant deformation retraction down to a copy of $S^{1}$, identified with

$$
S^{1}=\left\{(i x, i y) \mid(x, y) \in \mathbb{A}^{2}(\mathbb{R}), x^{2}+y^{2}=1\right\},
$$

and on this copy of $S^{1}, G$ acts by $(i x, i y) \mapsto(-i x,-i y)$, i.e., by the antipodal map, so $X$ is an algebraic model for $S^{0,2}$. The case of $W(\mathbb{C})$ is similar, but in this case the Galois action (if we identify $W(\mathbb{C})$ with $\mathbb{C}^{\times}$) is given by $z \mapsto \bar{z}^{-1}$, and $G$ acts trivially on

$$
S^{1}=\left\{(x, y) \mid(x, y) \in \mathbb{A}^{2}(\mathbb{R}), x^{2}+y^{2}=1\right\} .
$$

As shown in $[22$, Theorem A.1], $W(\mathbb{C})$ has a $G$-equivariant deformation retraction down to this copy of $S^{2,0}$, so $W$ is an algebraic model for $S^{2,0}$.

By [22], the algebraic $K$-theories of $W, X$, and $Y$ with finite coefficients in positive degrees thus coincide with $K R^{-\bullet}\left(S^{2,0}\right) \cong K O^{-\bullet} \oplus K O^{-\bullet-1}, K R^{-\bullet}\left(S^{0,2}\right) \cong$ $K S C^{-\bullet}$ and with $K R^{-\bullet}\left(S^{1,1}\right) \cong K O^{-\bullet} \oplus K O^{-\bullet+1}$, respectively.

We also want an algebraic model for $S_{+,-}^{1,1}$. This requires a noncommutative twisting of $Y$, given by an Azumaya algebra. Now it was shown in [10] that 
$\operatorname{Br} Y \cong(\mathbb{Z} / 2)^{2}$, since $Y(\mathbb{R})$ has two connected components (in the Hausdorff topology). In other words, there is an Azumaya algebra over $Y$ whose splitting over the two connected components of $Y(\mathbb{R})$ is whatever one would like. To get the sign choice $(+,-)$ over the two O-planes, we require an Azumaya algebra split over one component and non-split over the other. In fact, we can write this down very explicitly; let $\mathcal{A}=\mathbb{R}\left\langle t, t^{-1}, u\right\rangle /\left(u^{2}=-1\right.$, ut $\left.=-t u\right)$. This is a noncommutative Noetherian $\mathbb{R}$-algebra with center $Z(\mathcal{A})=\mathbb{R}\left[t^{2}, t^{-2}\right] \cong \mathbb{R}\left[v, v^{-1}\right]$, in fact a quaternion algebra over $Z(\mathcal{A})$ in the sense of [20], [33], or [44]. Over a point in $Y(\mathbb{R})$, corresponding to setting $t^{2} \mapsto \alpha \in \mathbb{R}^{\times}$, splitting of $\mathcal{A}$ is determined by the Hilbert symbol $(\alpha,-1)=\left\{\begin{array}{ll}+1, & \alpha>0 \\ -1, & \alpha<0\end{array}\right.$, and thus the algebra $\mathcal{A}$ does indeed correspond to the sign choice $(+,-)$. It follows that with finite coefficients, the algebraic $K$-theory of $\mathcal{A}$ in positive degrees agrees with $K R_{(+,-)}^{-\bullet}\left(S^{1,1}\right) \cong K S C^{1-\bullet}$.

2.2. The first duality. The first duality for orientifold theories on the circle relates the type I and IA theories, and is reflected in the $K R$-theory isomorphism $K R^{1-\bullet}\left(S^{2,0}\right)=K O^{1-\bullet}\left(S^{1}\right) \cong K R^{-\bullet}\left(S^{1,1}\right)$. We showed in [40] that this comes from the Baum-Connes isomorphism $K O_{\bullet}^{\text {top }}(B \mathbb{Z}) \stackrel{\cong}{\rightarrow} K O_{\bullet}^{\text {top }}\left(C_{\mathbb{R}}^{*}(\mathbb{Z})\right) \cong K R^{-\bullet}\left(S^{1,1}\right)$. Via the Karoubi-Weibel connection between $K R$ and algebraic $K$-theory of real varieties, this suggests comparing $K_{\bullet}(Y)$ and $K_{\bullet-1}(W)$. By the Fundamental Theorem of Algebraic $K$-Theory (recall that all of $W, X$, and $Y$ are smooth), we have

$$
K_{\bullet}(Y)=K_{\bullet}\left(\mathbb{R}\left[v, v^{-1}\right]\right) \cong K_{\bullet}(\mathbb{R}) \oplus K_{\bullet-1}(\mathbb{R}) .
$$

At the same time, we have

$$
K_{\bullet}(W)=K_{\bullet}\left(\mathbb{R}[x, y] /\left(x^{2}+y^{2}-1\right)\right)=K_{\bullet}(\mathbb{R}(q)),
$$

where $q(x, y)=x^{2}+y^{2}$, in the notation of [43], which computes the $K$-theory of quadric hypersurfaces. (In Swan's notation, $\mathbb{R}(q)$ is the quotient of $\mathbb{R}[x, y]$ by the ideal generated by $q(x, y)-1$.)

We certainly cannot have $K_{n}(Y) \cong K_{n-1}(W)$ when $n=0$, since $K_{0}(Y)=\mathbb{Z}$ and $K_{-1}(W)=0$. It's also known that $K_{0}(W) \cong \mathbb{Z} \oplus \mathbb{Z} / 2$ (there are many proofs, such as [43, Corollary 10.8 or $\S 10$, Example 3$]$, but for a completely elementary argument see $\left[39\right.$, Exercise 1.4.23]), while we see that $K_{1}(Y) \cong K_{1}(\mathbb{R}) \oplus K_{0}(\mathbb{R}) \cong \mathbb{R}^{\times} \oplus \mathbb{Z}$, which surjects onto $K_{0}(W) \cong \mathbb{Z} \oplus \mathbb{Z} / 2$ with completely divisible kernel.

For a complete calculation of $K_{\bullet}(W)$ we can use [43]. Note that $W$ is an open affine subscheme of the (Severi-Brauer) projective variety over $\mathbb{R}$ defined by the homogeneous equation $x^{2}+y^{2}-z^{2}=0$ in $\mathbb{P}^{2}$. By [45, Example V.1.6.1], this variety is simply $\mathbb{P}^{1}(\mathbb{R})$. The complement $\mathbb{P}^{1} \backslash W$ has no real points and two conjugate complex points; as a scheme it is just Spec $\mathbb{C}$. By Quillen's calculation of $K$-theory for projective spaces $\left([36, \S 8]\right.$ or $\left[45\right.$, Theorem V.1.5]), $K_{\bullet}\left(\mathbb{P}^{1}(\mathbb{R})\right) \cong$ $K_{\bullet}(\mathbb{R}) \oplus K_{\bullet}(\mathbb{R})$. Thus we derive the exact sequence (which also appears as $[43$, 
Theorem 2]):

$$
\cdots \rightarrow K_{n}(\mathbb{C}) \stackrel{\alpha}{\rightarrow} K_{n}(\mathbb{R}) \oplus K_{n}(\mathbb{R}) \rightarrow K_{n}(W) \stackrel{\partial}{\rightarrow} K_{n-1}(\mathbb{C}) \rightarrow \cdots
$$

Since $W(\mathbb{R}) \neq \emptyset$, one copy of $K_{n}(\mathbb{R})$ splits off from $K_{n}(W)$ and the complement $\widetilde{K}_{n}(W)$ is given by the homotopy groups of the cofiber $\widetilde{\mathbb{K}}(W)$ of a map $r: \mathbb{K}(\mathbb{C}) \rightarrow$ $\mathbb{K}(\mathbb{R})$. From the description in $[43$, Theorem 2] of the map $\alpha$ in (1) as a forgetful map, or from Quillen's description of the $K$-theory of projective space, one can see that $r$ is just the realification or transfer map obtained from viewing finitedimensional complex vector spaces as real vector spaces of twice the dimension.

Note by the way that the calculation of $K_{\bullet}(Y)$ could also be done by the same method as for $K_{\bullet}(W)$, as $Y$ is an open subscheme of the projective nonsingular curve defined by the homogeneous equation $x y-z^{2}=0$, which again is $\mathbb{P}^{1}(\mathbb{R})$. The complement $\mathbb{P}^{1} \backslash Y$ is Spec $\mathbb{R} \sqcup$ Spec $\mathbb{R}$, so the analogue of (1) is

$$
\cdots \rightarrow K_{n}(\mathbb{R}) \oplus K_{n}(\mathbb{R}) \stackrel{\alpha}{\rightarrow} K_{n}(\mathbb{R}) \oplus K_{n}(\mathbb{R}) \rightarrow K_{n}(Y) \stackrel{\partial}{\rightarrow} K_{n-1}(\mathbb{R}) \oplus K_{n-1}(\mathbb{R}) \cdots
$$

which after removing $K_{n}(\mathbb{R}) \stackrel{\cong}{\rightrightarrows} K_{n}(\mathbb{R})$ from $\alpha$ splits into split short exact sequences

$$
0 \rightarrow K_{n}(\mathbb{R}) \rightarrow K_{n}(Y) \rightleftarrows K_{n-1}(\mathbb{R}) \rightarrow 0 .
$$

The algebraic $K$-theory version of the T-duality between the type I and type IA theories on the circle now takes the following form.

Theorem 1. Let $W=\operatorname{Spec}\left(\mathbb{R}[x, y] /\left(x^{2}+y^{2}-1\right)\right)$ be the algebraic circle and let $Y=\mathbb{G}_{m}$ ( over $\left.\mathbb{R}\right)$. The $K$-theory spectrum of $Y$ splits as $\mathbb{K}(Y) \cong \mathbb{K}(\mathbb{R}) \oplus \Sigma \mathbb{K}(\mathbb{R})$. The $K$-theory spectrum of $W$ splits as $\mathbb{K}(W) \cong \mathbb{K}(\mathbb{R}) \oplus \widetilde{\mathbb{K}}(W)$, where $\widetilde{\mathbb{K}}(W)$ is the cofiber of the realification map $r: \mathbb{K}(\mathbb{C}) \rightarrow \mathbb{K}(\mathbb{R})$. There is a map of $K$-theory spectra with finite coefficients $\Omega \mathbb{K}(\mathbb{R} ; \mathbb{Z} / m) \rightarrow \widetilde{\mathbb{K}}(W ; \mathbb{Z} / m)$ for which the induced map on homotopy groups

$$
K_{n+1}(\mathbb{R} ; \mathbb{Z} / m) \rightarrow \widetilde{K}_{n}(W ; \mathbb{Z} / m)
$$

is an isomorphism for $n \geq 0$ and any $m>0$.

In lowest dimensions we have $K_{0}(Y) \cong \mathbb{Z}, K_{1}(Y) \cong \mathbb{R}^{\times} \oplus \mathbb{Z}, K_{2}(Y) \cong K_{2}(\mathbb{R}) \oplus$ $\mathbb{R}^{\times} \cong \mathbb{R}^{\times} \oplus \mathbb{Z} / 2 \oplus$ (uniquely divisible $), K_{0}(W) \cong \mathbb{Z} \oplus \mathbb{Z} / 2, K_{1}(W) \cong \mathbb{R}^{\times} \oplus \mathbb{Z} / 2$.

Proof. Most of this is contained in the above discussion, but it remains to relate the spaces $\Omega \mathbb{K}(\mathbb{R})$ and $\widetilde{\mathbb{K}}(W)$. Recall that by $[42]$, the natural maps $\mathbb{K}(\mathbb{R}) \rightarrow$ $\mathbb{K}^{\text {top }}(\mathbb{R})$ and $\mathbb{K}(\mathbb{C}) \rightarrow \mathbb{K}^{\text {top }}(\mathbb{C})$ are equivalences in nonnegative degrees with finite coefficients. Now by real Bott periodicity, there is a cofiber sequence of spectra

$$
\mathbb{K}^{\mathrm{top}}(\mathbb{R}) \stackrel{c}{\rightarrow} \mathbb{K}^{\mathrm{top}}(\mathbb{C}) \stackrel{r \circ \beta^{-1}}{\longrightarrow} \Sigma^{2} \mathbb{K}^{\mathrm{top}}(\mathbb{R})
$$

where $c$ is "complexification" and $\beta: \mathbb{K}^{\text {top }}(\mathbb{C}) \stackrel{\cong}{\rightarrow} \Omega^{2} \mathbb{K}^{\text {top }}(\mathbb{C})$ is the Bott map, associated to a familiar complexification/realification sequence in topological $K$ theory (see for example [6, Theorem 1.18(5)] or [21, Theorem III.5.18]). That 
means that the cofiber of $r: \mathbb{K}^{\text {top }}(\mathbb{C}) \rightarrow \mathbb{K}^{\text {top }}(\mathbb{R})$ can be identified with $\Omega \mathbb{K}^{\text {top }}(\mathbb{R})$. Because of Suslin's Theorem, the result follows.

Remark 2. One might wonder if there is some equivalence of derived categories underlying the $K$-theory isomorphism in Theorem 1 , but because of [37, Proposition 9.2], there are no nontrivial derived equivalences between affine schemes. The projective case is different, as we will see later.

2.3. The second duality. Now we want to look for an algebraic analogue of the duality between the $\widetilde{\mathrm{I}}$ and $\widetilde{\mathrm{IA}}$ theories on $S^{1}$. This means we want to relate the algebraic $K$-theories of $X$ and the Azumaya algebra $\mathcal{A}$ over $Y$.

To begin, we can compute the $K$-theory of $X=\mathbb{R}(q)$ with $q(x, y)=-x^{2}-y^{2}$ the same way we did for $W$. The major difference is that $X$ has no real points, nor does its projective completion, the quadric in $\mathbb{P}^{2}$ defined by $x^{2}+y^{2}+z^{2}=0$. Thus this quadric is a Brauer-Severi variety associated to a non-split Azumaya algebra, namely $\mathbb{H}$. So by Quillen's calculation in $[36, \S 8]$, the $K$-theory spectrum of this variety is $\mathbb{K}(\mathbb{R}) \oplus \mathbb{K}(\mathbb{H})$, and as before in (1) we get an exact sequence (which again could be read off from [43, Theorem 2])

$$
\cdots \rightarrow K_{n}(\mathbb{C}) \stackrel{\alpha}{\rightarrow} K_{n}(\mathbb{R}) \oplus K_{n}(\mathbb{H}) \rightarrow K_{n}(X) \stackrel{\partial}{\rightarrow} K_{n-1}(\mathbb{C}) \rightarrow \cdots
$$

Following [43] again, the map $\alpha$ comes from restriction of modules over Clifford algebras, from $M_{2}(\mathbb{C})$ to the subalgebras $\mathbb{H}$ and $M_{2}(\mathbb{R})$. The first component of $\alpha$ is $r: \mathbb{K}(\mathbb{C}) \rightarrow \mathbb{K}(\mathbb{R})$; the second component is the map $\mathbb{K}(\mathbb{C}) \rightarrow \mathbb{K}(\mathbb{H})$ induced by restriction of modules over $M_{2}(\mathbb{C})$ to $\mathbb{H}$, whose analogue in topological $K$-theory fits into a shifted version of (2). The lowest-degree portion of (3) looks like

$$
\cdots \rightarrow \mathbb{C}^{\times} \stackrel{\alpha}{\rightarrow} \mathbb{R}^{\times} \times\left(\mathbb{H}^{\times} / S U(2)\right) \rightarrow K_{1}(X) \rightarrow \mathbb{Z} \stackrel{a \mapsto(2 a, a)}{\rightarrow} \mathbb{Z}^{2} \rightarrow K_{0}(X) \rightarrow 0 .
$$

Thus $K_{0}(X) \cong \mathbb{Z}$ and $K_{1}(X) \cong \mathbb{R}^{\times}$. Note that modulo completely divisible abelian groups these agree with $K R^{-j}(X(\mathbb{C})) \cong K S C^{-j}$ for $j=0,1$, as we might expect from [22]. In fact, with finite coefficients and in positive degrees, we can replace algebraic $K$-theory by topological $K$-theory in (3) using [42], and then (3) becomes the known exact sequence [21, III.7.15]

$$
\cdots \rightarrow K S C^{-n-1} \rightarrow K^{-n} \rightarrow K O^{-n} \oplus K S p^{-n} \rightarrow K S C^{-n} \rightarrow \cdots .
$$

We turn now to the $K$-theory of the noncommutative algebra $\mathcal{A}$ from Section 2.1. In the notation of [43], this algebra is a Clifford algebra $C(q)$ over $R=\mathbb{R}\left[t^{2}, t^{-2}\right]$ attached to a rank-two quadratic $R$-module $(M, q)$, where if $e_{1}, e_{2}$ are an $R$-basis for the free $R$-module $M, q\left(e_{1}\right)=-1, q\left(e_{2}\right)=t^{2}$, and $e_{1} \perp e_{2}$. Since $t^{2}$ is a unit in $R, q$ is non-singular. Furthermore, $C(q) \cong C_{0}(q \oplus\langle-1\rangle)$ by [43, Lemma 4.5]. Thus we can apply [43, Theorem 9.1] with $d=1$ to get $\mathbb{K}(\mathcal{A}) \oplus \mathbb{K}(R) \cong \mathbb{K}(Z)$. Here $Z=X(q \oplus\langle-1\rangle)$ is the closed subscheme of $\mathbb{P}^{2}(R)$ defined by the homogeneous equation $q \oplus\langle-1\rangle=0$. Alternatively, by $[36, \S 8$, Theorem 4.1], $\mathbb{K}(\mathcal{A}) \oplus \mathbb{K}(R) \cong$ $\mathbb{K}(Z)$, where $Z$ the Brauer-Severi scheme over $Y$ associated to $\mathcal{A}$, and this turns 
out to be the same as above. So we're basically reduced to computing the $K$-theory of $Z$.

This is still a formidable task, so it is easiest to take another approach and to regard $\mathcal{A}$ as a crossed product or twisted Laurent polynomial extension $\mathbb{C} \rtimes_{\sigma} \mathbb{Z}=$ $\mathbb{C}_{\sigma}\left[t, t^{-1}\right]$, where $\mathbb{C}=\mathbb{R}[u] /\left(u^{2}+1\right)$ and $\sigma$ is complex conjugation (since conjugation by $t$ sends $u$ to $-u$ ). Thus by the "algebraic Pimsner-Voiculescu sequence" of $[15$, Theorems 2.1 and 2.3] or [49, Corollary 2.2] (Nil terms drop out since everything is regular $), \mathbb{K}(\mathcal{A})$ is equivalent to the cofiber of $\mathbb{K}(\mathbb{C}) \stackrel{1-\sigma_{*}}{\longrightarrow} \mathbb{K}(\mathbb{C})$. In particular, in low degrees we get

$$
\cdots \rightarrow \star \rightarrow \star \rightarrow K_{2}(\mathcal{A}) \rightarrow \mathbb{C}^{\times} \stackrel{z \mapsto z / \bar{z}}{\longrightarrow} \mathbb{C}^{\times} \rightarrow K_{1}(\mathcal{A}) \rightarrow \mathbb{Z} \stackrel{0}{\rightarrow} \mathbb{Z} \rightarrow K_{0}(\mathcal{A}) \rightarrow 0,
$$

where $\star=K_{2}(\mathbb{C})$ is uniquely divisible, so $K_{2}(\mathcal{A}) \cong \mathbb{Z} / 2 \oplus$ (uniquely divisible), $K_{1}(\mathcal{A}) \cong \mathbb{R}_{+}^{\times} \oplus \mathbb{Z}$ and $K_{0}(\mathcal{A}) \cong \mathbb{Z}$.

The algebraic $K$-theory version of the T-duality between the $\widetilde{\mathrm{I}}$ and $\widetilde{\mathrm{IA}}$ theories on the circle now takes the following form.

Theorem 3. Let $X=\operatorname{Spec}\left(\mathbb{R}[x, y] /\left(x^{2}+y^{2}+1\right)\right)$, a smooth affine quadric curve with no real points, and let $\mathcal{A}$ be the quaternion algebra over $Y=\mathbb{G}_{m}$ given by $\mathcal{A}=\mathbb{R}\left\langle t, t^{-1}, u\right\rangle /\left(u^{2}=-1, u t=-t u\right)$. The $K$-theory spectrum of $\mathcal{A}$ is the cofiber of $\mathbb{K}(\mathbb{C}) \stackrel{1-\sigma_{*}}{\longrightarrow} \mathbb{K}(\mathbb{C})$, where $\sigma$ is complex conjugation. The $K$-theory spectrum of $X$ is the cofiber of $\mathbb{K}(\mathbb{C}) \stackrel{\alpha}{\rightarrow} \mathbb{K}(\mathbb{R}) \oplus \mathbb{K}(\mathbb{H})$, where $\alpha$ comes from restriction of Clifford modules, as explained above. There is a map of $K$-theory spectra with finite coefficients $\Omega \mathbb{K}(\mathcal{A} ; \mathbb{Z} / m) \rightarrow \mathbb{K}(X ; \mathbb{Z} / m)$ for which the induced map on homotopy groups

$$
K_{n+1}(\mathcal{A} ; \mathbb{Z} / m) \rightarrow K_{n}(X ; \mathbb{Z} / m)
$$

is an isomorphism for $n \geq 0$ and any $m>0$.

In lowest dimensions we have $K_{0}(X) \cong \mathbb{Z}, K_{1}(X) \cong \mathbb{R}^{\times}, K_{0}(\mathcal{A}) \cong \mathbb{Z}, K_{1}(\mathcal{A}) \cong$ $\mathbb{R}_{+}^{\times} \oplus \mathbb{Z}$.

Proof. Again, most of this is in the discussion above. After applying [42] and going to the stable range (so we can ignore the difference between connective and periodic $K$-theory spectra), we see that with finite coefficients, $\mathbb{K}(\mathcal{A})$ becomes the cofiber of $\mathbb{K}^{\text {top }}(\mathbb{C}) \stackrel{1-\sigma_{*}}{\longrightarrow} \mathbb{K}^{\text {top }}(\mathbb{C})$, which is $\Sigma \mathbb{K S C}, \mathbb{K} \mathbb{S} C$ the 4-periodic spectrum of self-conjugate $K$-theory. Similarly $\mathbb{K}(X)$ becomes the cofiber of $\mathbb{K}^{\text {top }}(\mathbb{C}) \stackrel{r}{\rightarrow}$ $\mathbb{K}^{\text {top }}(\mathbb{R}) \oplus \mathbb{K}^{\text {top }}(\mathbb{H})$, which is $\mathbb{K} \mathbb{S} \mathbb{C}$, and the result follows.

\section{Some Elliptic CURVE ORIENTIFOldS}

Now we consider cases of duality related to elliptic curves. Generally speaking, a duality between a type IIA theory and a type IIB theory on elliptic curves should in the world of algebraic mirror symmetry be reflected in an equivalence between a category of coherent sheaves on one side and a Fukaya category on the other [35]. Since the analogue of Fukaya categories in the orientifold case is not well 
developed yet, we leave such dualities as a project for future work. But there is one interesting case of an orientifold duality that we should be able to treat without going out of categories of coherent sheaves. This involves the T-duality grouping $[13, \S 4.1 .2]$ containing the type IIA theory on an elliptic curve with free antiholomorphic involution and the type IIA theory on an elliptic curve with antiholomorphic involution with exactly two fixed circles, one given a + charge and one given a - charge (the $\widetilde{\mathrm{IA}}$ theory). Since an elliptic curve with antiholomorphic involution is the same thing as a smooth projective genus-1 curve defined over $\mathbb{R}$, there are algebraic models for these theories involving coherent sheaves on real varieties, though in one case we need to twist by a suitable Azumaya algebra. That such an Azumaya algebra exists is guaranteed by an application of [9, Theorem 3.6], as indicated in the following Proposition. Note that in what follows, $\mathcal{A}$ represents a different Azumaya algebra than in Section 2 above. We recall that a smooth projective curve defined over $\mathbb{R}$ has an invariant called the species, which is the number of connected components of the set of real points (in the analytic topology). This invariant can take any value from 0 up to $g+1$ ( $g$ the genus), by a classical theorem of Harnack (see also [17, Proposition 3.1(1)]).

Proposition 4. Let $E$ be a split elliptic curve defined over $\mathbb{R}$ (i.e., in the language of [1], a real elliptic curve of species 2 , so that $E(\mathbb{R})$ is topologically a disjoint union of two circles). Then there is a unique (up to Morita equivalence) Azumaya algebra $\mathcal{A}$ over $E$ representing an element of $\operatorname{Br}(E)$ of order 2 , such that $\mathcal{A}$ is split over the component $E(\mathbb{R})^{+}$of $E(\mathbb{R})$ containing the identity element $e$ (for the group structure on $E(\mathbb{C})$ ) and non-split over the other component $E(\mathbb{R})^{-}$of $E(\mathbb{R})$.

Proof. A split elliptic curve defined over $\mathbb{R}$ has Weierstraß equation

$$
y^{2}=(x-\alpha)(x-\beta)(x-\gamma),
$$

with $\alpha<\beta<\gamma$ distinct points in $\mathbb{R}$. The 2-torsion subgroup $M={ }_{2} E(\mathbb{C})$ of $E(\mathbb{C})$ consists of $e$ (the point at $\infty$ ) and the points $a=(\alpha, 0), b=(\beta, 0)$, and $c=(\gamma, 0)$, with $c$ and $e$ in one component of $E(\mathbb{R})$, which we'll call $E(\mathbb{R})^{+}$, and $a$ and $b$ in the other component $E(\mathbb{R})^{-}$. Let $G=\operatorname{Gal}(\mathbb{C} / \mathbb{R})$. By $[34$, Proposition 3.6], $\operatorname{Br}(E) \cong(\mathbb{Z} / 2)^{2}$; in fact, as Pedrini and Weibel point out, this was really computed by Witt in [46, $\mathrm{II}^{\prime}$ and $\left.\mathrm{III}^{\prime}\right]$. More precisely, via an analysis of the $G$-action on the exact sequence

$$
0 \rightarrow M \rightarrow E(\mathbb{C}) \stackrel{2}{\rightarrow} E(\mathbb{C}) \rightarrow 0,
$$

it is shown in $[9]$ that ${ }_{2} \mathrm{Br} E={ }_{2} \mathrm{Br} \mathbb{R} \oplus_{2} H^{1}(G, E(\mathbb{C}))$. As a $G$-module, $E(\mathbb{C})$ looks like $(\mathbb{T}$, id $) \times(\mathbb{T}, \sigma)$, where $\mathbb{T}$ as usual is the circle group, id is the trivial $G$-action, and $\sigma$ is complex conjugation. So

$$
H^{1}(G, E(\mathbb{C}))=\frac{\operatorname{ker}\left((z, w) \mapsto(z, w)\left(z, w^{-1}\right)\right)}{\operatorname{image}\left((z, w) \mapsto(z, w)\left(z^{-1}, w\right)\right)} \cong \mathbb{Z} / 2,
$$


and ${ }_{2} \mathrm{Br} E$ is exactly the group of "sign choices" discussed in [12] and [27]. Thus there is a unique Brauer class of Azumaya algebras $\mathcal{A}$ split over $E(\mathbb{R})^{+}$and nonsplit over $E(\mathbb{R})^{-}$, which can be chosen to be represented by the quaternion algebra over the function field $\mathbb{R}(E)$ given by the quaternion algebra symbol $(-1, x-\gamma)$. (Note that $x-\gamma$ is positive on $E(\mathbb{R})^{+}$, so the Hilbert symbol there is trivial, while it is negative on $E(\mathbb{R})^{-}$, so the Hilbert symbol there is nontrivial.) Viewed as a sheaf of $\mathcal{O}_{E}$-modules, $\mathcal{A}$ is locally free of rank 4 , and its complexification is isomorphic to $M_{2}\left(\mathcal{O}_{E_{\mathbb{C}}}\right)$.

Now we are ready for what is really the main theorem of this paper. It says that in some sense, the "dual abelian variety" to a genus- 1 curve over $\mathbb{R}$ with no real points is the noncommutative Azumaya algebra of Proposition 4.

Theorem 5. Let $C$ be a smooth projective genus-1 curve over $\mathbb{R}$ with no real points (i.e., in the terminology of [1], a non-classical real elliptic curve of species $0)$. Let $E$ be the split elliptic curve over $\mathbb{R}$ with the same $j$-invariant. Let $\mathcal{A}$ be the Azumaya algebra over $E$ as identified in Proposition 4. Let $\mathcal{D}(C)$ be the bounded derived category of (complexes of) coherent sheaves on $C$, and let $\mathcal{D}(E, \alpha)$ be the bounded derived category of (complexes of) $\alpha$-twisted coherent sheaves on $E$, where $\alpha$ is the Brauer group class of $\mathcal{A}$. Then the categories $\mathcal{D}(C)$ and $\mathcal{D}(E, \alpha)$ are equivalent (in a way we will make explicit), and in particular, $K_{\bullet}(C) \cong K_{\bullet}(\mathcal{A})$.

Since there are several steps to the proof, we separate out the first part as a lemma.

Lemma 6. Let $C$ and $E$ be as in Theorem 5. Then the Jacobian variety of $C$ can be identified with $E$, and $\operatorname{Pic}^{0}(C)$ (the group of classes of $\mathrm{Gal}(\mathbb{C} / \mathbb{R})$-fixed divisors on $C$ of degree zero) can be identified with the connected component of the identity $E(\mathbb{R})^{+}$(which has index 2 ) in the group $E(\mathbb{R})$ of real points of $E$.

Proof of Lemma. As before we let $G=\operatorname{Gal}(\mathbb{C} / \mathbb{R})$. We begin by recalling a number of facts from [17] and [34] about the Picard group of $C$. First of all, $C_{\mathbb{C}}=C \times_{\operatorname{Spec} \mathbb{R}}$ Spec $\mathbb{C}$ is a complex elliptic curve and as such is self-dual. So the Picard group $\operatorname{Pic}\left(C_{\mathbb{C}}\right)$ of $C_{\mathbb{C}}$ can be identified with $\operatorname{Pic}^{0}\left(C_{\mathbb{C}}\right) \times \mathbb{Z}$, with $\operatorname{Pic}^{0}\left(C_{\mathbb{C}}\right) \cong C_{\mathbb{C}}$. As shown in [17, Proposition 2.2] and [34, Proposition 1.1], the Picard group $\operatorname{Pic}(C)$ of $C$ is a subgroup of $\operatorname{Pic}\left(C_{\mathbb{C}}\right)$, but has two unusual features. First of all, the image of $\operatorname{Pic}(C)$ in $\operatorname{Pic}\left(C_{\mathbb{C}}\right) / \operatorname{Pic}^{0}\left(C_{\mathbb{C}}\right) \cong \mathbb{Z}$ is of index two, i.e., all algebraic line bundles over $C$ (defined over $\mathbb{R}$ ) have even degree. Secondly, $\left(\operatorname{Pic}^{0}\left(C_{\mathbb{C}}\right)\right)^{G}$ contains the connected component of the identity $\operatorname{Pic}^{0}(C)$ in $\operatorname{Pic}(C)$ with index 2. The connected component of the identity, denoted $\mathrm{Pic}^{+}(\mathbb{R})$ in $[17]$, consists of classes of $G$-fixed divisors, but there exist divisors whose class modulo rational equivalence is fixed by $G$ without the divisor itself being fixed by $G$. The upshot of this discussion is that the Jacobian variety of $C$ is a real elliptic curve of species 2 . It has to complexify to $C_{\mathbb{C}}$, so it has the same $j$-invariant as $C$, and can be identified with $E$, with $\operatorname{Pic}^{0}(C)$ identified with $E(\mathbb{R})^{+}$. In fact, there is an étale morphism $\pi$ 
(an isogeny) from $C$ onto $E$, which on the complexification $C_{\mathbb{C}}$ is just multiplication by 2. Complex analytically, $C$ can be identified with $\mathbb{C} /(\mathbb{Z}+\tau \mathbb{Z})$, where $\tau=i \tau_{0}$ with $\tau_{0}>0$, and the action of $G$ on $C$ is given by $\widetilde{\sigma}: z \mapsto \bar{z}+\frac{1}{2}$. (See for example $[1$, Chapter 12] and [13, §4.1.2] for the physical interpretation.) The map $\pi: z \mapsto 2 z$ intertwines this $G$-action with the $G$-action $\sigma: z \mapsto \bar{z}$ on the same torus, which corresponds to the species 2 curve $E$. In purely algebraic language, following the notation in the proof of Proposition 4, let $M=\{e, a, b, c\}$ be the 2-torsion subgroup of $E_{\mathbb{C}}$, where $e$ is the identity element (corresponding to the point at infinity in the Weierstraß form) and $e, c \in E(\mathbb{R})^{+}, a, b \in E(\mathbb{R})^{-}$. Then the Galois involution $\sigma$ on $E_{\mathbb{C}}$ fixes $M$ (pointwise), and the Galois involution $\widetilde{\sigma}$ on $C_{\mathbb{C}}$ is given by $z \mapsto \sigma(z)+c$. The map from $C_{\mathbb{C}}$ to its Jacobian can be identified with $\phi: z \mapsto T_{z}^{*} \mathcal{L} \otimes \mathcal{L}^{-1}$ (see [29, Chapter II]), where $T_{z}$ is translation by $z$ and $\mathcal{L}$ is the line bundle of degree 2 defined by the divisor $[e]+[c]$. This divisor is $\widetilde{\sigma}$-invariant, so that $\mathcal{L}$ is defined over $\mathbb{R}$, as is $\phi(z)$ for $z \in E(\mathbb{R})$. Note that the kernel of $\phi$ can be seen to be $M$, so again we see that the Jacobian of $C$ is $C / M \cong E$, and $\phi$ can be identified with $\pi: C \rightarrow E$.

Proof of Theorem 5. Recall from [7, Theorem 1.3.5] that we may identify $\mathcal{A}$ with the endomorphism algebra bundle of $\mathcal{E}$, where $\mathcal{E}$ is a rank- $2 \alpha$-twisted locally free sheaf over $E$. (We view $\alpha$ as a class in $H_{\text {an }}^{2}\left(E, \mathcal{O}_{E}^{\times}\right)$, cohomology for the analytic topology; cf. [7, Theorem 1.1.4].) Furthermore, there is an equivalence of categories between coherent $\mathcal{A}$-modules and coherent $\alpha$-twisted sheaves over $E$, which we will use hereafter without special comment.

With these preliminaries out of the way, we now define a twisted bundle $\mathcal{P}$ on $C \times E$, playing the same role as the Poincaré bundle in Mukai duality [28], that will implement the derived equivalence $\mathcal{D}(C) \cong \mathcal{D}(\mathcal{A}) \cong \mathcal{D}(E, \alpha)$, following the paradigm in $[31,32]$ and $[7,8]$. Identify $C_{\mathbb{C}}$ and $E_{\mathbb{C}}$ as usual and let $\mathcal{P}_{0}$ be the Poincaré bundle on $C_{\mathbb{C}} \times E_{\mathbb{C}}$, which makes sense since $E_{\mathbb{C}}$ is the Jacobian variety of $C_{\mathbb{C}}$. In other words, for $x \in E_{\mathbb{C}}$, the restriction of $\mathcal{P}_{0}$ to $C_{\mathbb{C}} \times\{x\}$ is the line bundle $\mathcal{V}_{x}$ defined by $x$ over $C_{\mathbb{C}}$. According to the the recipe in [29, Chapter II, $\S 8], \mathcal{P}_{0}$ is defined by the divisor

$$
D_{0}=[\Delta]-\left[C_{\mathbb{C}} \times\{e\}\right]-\left[\{e\} \times E_{\mathbb{C}}\right]
$$

where $\Delta$ is the anti-diagonal $\left\{(x,-x): x \in C_{\mathbb{C}}\right\}$. Note that $\Delta$ can also be described as $m^{-1}(e)$, where $m$ is the additive group law on $C_{\mathbb{C}}=E_{\mathbb{C}}$. Now $\sigma \times \sigma$ obviously maps $\Delta$ to itself, so the Galois involution $\tilde{\sigma} \times \sigma$ on $C_{\mathbb{C}} \times E_{\mathbb{C}}$ sends $\Delta$ to $\Delta+(c, e)$ and vice versa. Similarly, $\widetilde{\sigma} \times \sigma$ maps $C_{\mathbb{C}} \times\{e\}$ to itself, and interchanges $\{e\} \times E_{\mathbb{C}}$ and $\{c\} \times E_{\mathbb{C}}$. So the divisor $D_{0}$ is not invariant under $G$ and the conjugate bundle $\overline{\mathcal{P}_{0}}$ is associated to the divisor

$$
(\widetilde{\sigma} \times \sigma)\left(D_{0}\right)=[\Delta+(c, e)]-\left[C_{\mathbb{C}} \times\{e\}\right]-\left[\{c\} \times E_{\mathbb{C}}\right] .
$$

Let $\mathcal{P}=\mathcal{P}_{0} \oplus \overline{\mathcal{P}_{0}}$. Even though the individual summands $\mathcal{P}_{0}$ and $\overline{\mathcal{P}_{0}}$ are not defined over $\mathbb{R}, \mathcal{P}$ is defined over $\mathbb{R}$, with $G$ interchanging the two summands. 
For $x \in E_{\mathbb{C}}$, the restriction of $\mathcal{P}$ to $C_{\mathbb{C}} \times\{x\}$ is $\mathcal{V}_{x} \oplus \overline{\mathcal{V}_{x}}$, where $\mathcal{V}_{x}$ defined by the element $x$ of the Jacobian of $C_{\mathbb{C}}$. In other words, $\mathcal{V}_{x}=\phi(z)=T_{z}^{*} \mathcal{L} \otimes \mathcal{L}^{-1}$, where $2 z=-x$ and $z$ is uniquely defined modulo $M$. Since $\mathcal{P}$ is defined over $\mathbb{R}$, it is an $\mathcal{O}_{C \times E}$-module. (Note that $\mathcal{O}_{C \times E}=\mathcal{O}_{C} \otimes \mathcal{O}_{E}$, the external tensor product.) We just need to explain how to make $\mathcal{P}$ into an $\mathcal{O}_{C} \otimes \mathcal{A}$-module. Since $\mathcal{P}=\mathcal{P}_{0} \oplus \overline{\mathcal{P}_{0}}$, multiplication by $i$ on $\mathcal{P}$ acts by the matrix $\mathcal{I}=\left(\begin{array}{cc}i & 0 \\ 0 & -i\end{array}\right)$. Recall that the gerbe $\alpha$ defining $\mathcal{A}$ is trivial on $E(\mathbb{R})^{+}$but nontrivial on $E(\mathbb{R})^{-}$. At the same time, for $x \in E(\mathbb{R})^{+}$, the associated line bundle $\mathcal{V}_{x}$ is defined over $\mathbb{R}$. (The divisor of $\mathcal{V}_{x}$ is $\mu=D_{0} \cap\left(C_{\mathbb{C}} \times\{x\}\right)=[-x]-[e]$. This divisor is not $G$-invariant, but it's rationally equivalent to $[z]+[z+c]-[e]-[c]$, which is $G$ invariant. Here we choose $z \in E(\mathbb{R})$ with $2 z=-x$, possible since $x \in E(\mathbb{R})^{+}$.) So we get a vector bundle isomorphism $\mathcal{V}_{x} \rightarrow \overline{\mathcal{V}_{x}}$ and thus an endomorphism $J$ of $\mathcal{V}_{x} \oplus \overline{\mathcal{V}_{x}}$ anticommuting with $\mathcal{I}$ and with $J^{2}=1$. Since $\mathcal{I}$ and $J$ satisfy the relations for a split quaternion algebra over $\mathbb{R}, C_{\mathbb{C}} \times E(\mathbb{R})^{+}$is a module for $\left(\mathcal{O}_{E} \otimes \mathcal{A}\right) \cong\left(\mathcal{O}_{E} \otimes M_{2}\left(\mathcal{O}_{C}\right)\right)$ over $C_{\mathbb{C}} \times E(\mathbb{R})^{+}$. For $x \in E(\mathbb{R})^{-}$, the associated line bundle $\mathcal{V}_{x}$ is not defined over $\mathbb{R}$, but as explained in the proof of $[17$, Proposition $2.2]$, there is an isomorphism $\varphi_{x}: \mathcal{V}_{x} \rightarrow \overline{\mathcal{V}_{x}}$ with $\varphi_{x}^{2}=-1$. (In other words, we get an endomorphism $J$ anticommuting with $\mathcal{I}$ and with $J^{2}=-1$.) This gives $\mathcal{V}_{x} \oplus \overline{\mathcal{V}_{x}}$ a quaternionic structure. In fact (after renormalizing) we can make this structure algebraic in the variable $x$. To see this, again change the divisor $\mu$ of $\mathcal{V}_{x}$ up to rational equivalence to the form $\mu^{\prime}=[z]+[z+c]-[e]-[c]$ with $2 z=-x$. Since $x \in E(\mathbb{R})^{-}, z$ cannot be chosen in $E(\mathbb{R})$. But $\widetilde{\sigma}\left(\mu^{\prime}\right)=[\sigma(z)+c]+[\sigma(z)]-[c]-[e]$, and $\mu-\widetilde{\sigma}(\mu)=[z]-[\sigma(z)]+[z+c]-[\sigma(z)+c]$ is a principal divisor, in fact associated to the meromorphic function $f: w \mapsto \operatorname{sn}(w-z)$, where sn is the standard Jacobi elliptic function with parameters $K=\frac{1}{4}, K^{\prime}=\frac{i \tau_{0}}{2}[11, \S 22.4]$. This is obviously meromorphic in $z$ and thus in $x$, and as indicated in [17], multiplication by this function implements the quaternionic structure. Indeed, $w \mapsto \overline{\operatorname{sn}(\widetilde{\sigma}(w)-z)}$ is also meromorphic in $w$, and taking $w=z+K$ and using the fact that $\bar{z}-z=-i K^{\prime}$ (since $2 z \in E(\mathbb{R})^{-}$),

$$
\begin{aligned}
\operatorname{sn}(w-z) & =\operatorname{sn}(K)=1, \\
\overline{\operatorname{sn}(\widetilde{\sigma}(w)-z)} & =\overline{\operatorname{sn}(\bar{w}+2 K-z)} \\
& =\overline{\operatorname{sn}\left(3 K-i K^{\prime}\right)}=-\overline{\operatorname{sn}\left(K+i K^{\prime}\right)}=\frac{-1}{k}<0 .
\end{aligned}
$$

(The negative sign of the product, what is called $\mathbf{N} f$ in [17], is what counts here.) Thus $f$ makes the restriction of $\mathcal{P}$ to $C_{\mathbb{C}} \times E(\mathbb{R})^{-}$into a module for $\left(\mathcal{O}_{E} \otimes \mathcal{A}\right) \cong$ $\left(\mathcal{O}_{E} \otimes \mathcal{O}_{C} \otimes \mathbb{H}\right)$ over $C_{\mathbb{C}} \times E(\mathbb{R})^{-}$. These $\mathcal{O}_{E} \otimes \mathcal{A}$-module structures on $\mathcal{P}$ extend to open neighborhoods of $C_{\mathbb{C}} \times E(\mathbb{R})^{+}$and $C_{\mathbb{C}} \times E(\mathbb{R})^{-}$(in the analytic topology) which, together, cover $C_{\mathbb{C}} \times E_{\mathbb{C}}$. Patching these together, we obtain an $\alpha$-twisted 
sheaf structure on $\mathcal{P}$ over $C \times E$. By the usual process, we obtain a functor

$$
\Phi: \mathcal{D}(C) \rightarrow \mathcal{D}(\mathcal{A}), \quad \Phi(\mathcal{F})=\mathbf{R}\left(\pi_{E}\right)_{*}\left(\mathcal{P} \stackrel{\mathbf{L}}{\otimes} \mathbf{L}\left(\pi_{C}\right)^{*} \mathcal{F}\right),
$$

and a similar functor $\Psi: \mathcal{D}(\mathcal{A}) \rightarrow \mathcal{D}(C)$ obtained by reversing the roles of $E$ and $C$.

We can now prove the theorem by using [31, Lemma 2.12], modified in the obvious way to deal with twisted sheaves. This asserts that the property of $\Phi$ being an equivalence is stable under base change from $\mathbb{R}$ to $\mathbb{C}$. But the complexification $\mathcal{A}_{\mathbb{C}}$ of $\mathcal{A}$ is $M_{2}\left(\mathcal{O}_{E_{\mathbb{C}}}\right)$, Morita equivalent to $\mathcal{O}_{E_{\mathbb{C}}}$, so $\mathcal{D}\left(\mathcal{A}_{\mathbb{C}}\right)$ is equivalent to $\mathcal{D}\left(E_{\mathbb{C}}\right)$. Hence $\Phi$ is an equivalence because the functor $\Phi_{\mathbb{C}}: \mathcal{D}\left(E_{\mathbb{C}}\right) \rightarrow \mathcal{D}\left(C_{\mathbb{C}}\right)$ is the equivalence given by Mukai duality [28].

Remark 7. To link Theorem 5 to the results of [8] and [3], note that our genus-1 curve $C$ can be viewed as an elliptic fibration over $\operatorname{Spec} \mathbb{R}$ with Jacobian $E$, but without a section, and also as a torsor over the elliptic curve $E$. To see this, consider the translation action $m$ of $E_{\mathbb{C}}$ on $C_{\mathbb{C}}$ (say on the right): $C_{\mathbb{C}} \times E_{\mathbb{C}} \stackrel{m}{\rightarrow} C_{\mathbb{C}}$, $m(y, x)=y+x$. This action is equivariant for the Galois action, since

$m((\widetilde{\sigma} \times \sigma)(y, x))=m(\sigma(y)+c, \sigma(x))=\sigma(y)+c+\sigma(x)=\sigma(y+x)+c=\widetilde{\sigma}(m(y, x))$.

Thus we can think of $C$ as a principal homogeneous space for $E$. In [3], derived equivalences of Brauer twists for principal homogeneous spaces over an elliptic curve are studied, but Theorem 5.1 in that paper, for example, only deals with the case where the Brauer twist comes from the Brauer group of the field, Br $\mathbb{R}$ in our case. In our situation, $E$ is of course a trivial torsor over itself, while $C$ has a nontrivial class in $H_{\text {ét }}^{1}(\operatorname{Spec} \mathbb{R}, E)=H^{1}(G, E(\mathbb{C})) \cong \mathbb{Z} / 2$. (Recall the proof of Proposition 4.) So our twist $\alpha$ comes from the cokernel of the map $\mathrm{Br} \mathbb{R} \rightarrow \operatorname{Br} E$, which in the language of Ogg-Shafarevich theory (see [8, §6]) is the Tate-Shafarevich group $\amalg_{\text {Spec } \mathbb{R}}(E) \cong \mathbb{Z} / 2$. Thus it seems that Theorem 5 is covered neither by the hypotheses in [3] nor by the hypotheses of [8], which only deals with algebraically closed fields. Nevertheless, the proof of [8, Theorem 5.1] could be adapted to give another proof of Theorem 5, using the étale topology on the base Spec $\mathbb{R}$.

\section{REFERENCES}

[1] Norman L. Alling. Real elliptic curves, volume 54 of North-Holland Mathematics Studies. North-Holland Publishing Co., Amsterdam, 1981. Notas de Matemática [Mathematical Notes], 81.

[2] D. W. Anderson. The real K-theory of classifying spaces. Proc. Nat. Acad. Sci. U. S. A., 51(4):634-636, 1964.

[3] B. Antieau, D. Krashen, and M. Ward. Derived categories of torsors for abelian schemes. arXiv:1409.2580, 2014.

[4] M. F. Atiyah. K-theory and reality. Quart. J. Math. Oxford Ser. (2), 17:367-386, 1966.

[5] Paul Baum and Max Karoubi. On the Baum-Connes conjecture in the real case. Q. J. Math., 55(3):231-235, 2004. 
[6] Jeffrey L. Boersema. Real $C^{*}$-algebras, united $K$-theory, and the Künneth formula. $K$ Theory, 26(4):345-402, 2002.

[7] Andrei Căldăraru. Derived categories of twisted sheaves on Calabi-Yau manifolds. ProQuest LLC, Ann Arbor, MI, 2000. Thesis (Ph.D.)-Cornell University.

[8] Andrei Căldăraru. Derived categories of twisted sheaves on elliptic threefolds. J. Reine Angew. Math., 544:161-179, 2002.

[9] V. Chernousov and V. Guletskiǔ. 2-torsion of the Brauer group of an elliptic curve: generators and relations. Doc. Math., extra volume, 2001:85-120 (electronic), 2001. Proceedings of the Conference on Quadratic Forms and Related Topics (Baton Rouge, LA, 2001).

[10] F. R. DeMeyer and M. A. Knus. The Brauer group of a real curve. Proc. Amer. Math. Soc., $57(2): 227-232,1976$.

[11] NIST Digital Library of Mathematical Functions. http://dlmf .nist.gov/, Release 1.0.10 of 2015-08-07. Online companion to [30].

[12] Charles Doran, Stefan Mendez-Diez, and Jonathan Rosenberg. T-duality for orientifolds and twisted KR-theory. Lett. Math. Phys., 104(11):1333-1364, 2014.

[13] Charles Doran, Stefan Mendez-Diez, and Jonathan Rosenberg. String theory on elliptic curve orientifolds and KR-theory. Comm. Math. Phys., 335(2):955-1001, 2015.

[14] Dongfeng Gao and Kentaro Hori. On the structure of the Chan-Paton factors for D-branes in type II orientifolds. preprint, 2010.

[15] Daniel R. Grayson. The K-theory of semilinear endomorphisms. J. Algebra, 113(2):358-372, 1988.

[16] Paul S. Green. A cohomology theory based upon self-conjugacies of complex vector bundles. Bull. Amer. Math. Soc., 70:522-524, 1964.

[17] Benedict H. Gross and Joe Harris. Real algebraic curves. Ann. Sci. École Norm. Sup. (4), 14(2):157-182, 1981.

[18] Sergei Gukov. K-theory, reality, and orientifolds. Comm. Math. Phys., 210:621-639, 2000.

[19] Kentaro Hori. D-branes, T duality, and index theory. Adv. Theor. Math. Phys., 3:281-342, 1999.

[20] Teruo Kanzaki. Note on quaternion algebras over a commutative ring. Osaka J. Math., 13(3):503-512, 1976.

[21] Max Karoubi. K-theory: An introduction. Springer-Verlag, Berlin, 1978. Grundlehren der Mathematischen Wissenschaften, Band 226.

[22] Max Karoubi and Charles Weibel. Algebraic and Real $K$-theory of real varieties. Topology, 42(4):715-742, 2003.

[23] Dirk Kussin. Weighted noncommutative regular projective curves. arXiv:1412.0290, 2014.

[24] H. Blaine Lawson, Jr. and Marie-Louise Michelsohn. Spin geometry, volume 38 of Princeton Mathematical Series. Princeton University Press, Princeton, NJ, 1989.

[25] E.-K. M. Moutuou. Twistings of $K R$ for Real groupoids. preprint, 2011.

[26] E.-K. M. Moutuou. Twisted groupoid KR-theory. PhD thesis, Université de Lorraine, 2012. available at http://www.theses.fr/2012LORR0042.

[27] E.-K. M. Moutuou. Graded Brauer groups of a groupoid with involution. J. Funct. Anal., 266(5):2689-2739, 2014.

[28] Shigeru Mukai. Duality between $D(X)$ and $D(\widehat{X})$ with its application to Picard sheaves. Nagoya Math. J., 81:153-175, 1981.

[29] David Mumford. Abelian varieties, volume 5 of Tata Institute of Fundamental Research Studies in Mathematics. Published for the Tata Institute of Fundamental Research, Bombay, by Hindustan Book Agency, New Delhi, 2008. With appendices by C. P. Ramanujam and Yuri Manin, Corrected reprint of the second (1974) edition. 
[30] F. W. J. Olver, D. W. Lozier, R. F. Boisvert, and C. W. Clark, editors. NIST Handbook of Mathematical Functions. Cambridge University Press, New York, NY, 2010. Print companion to [11].

[31] D. O. Orlov. Derived categories of coherent sheaves on abelian varieties and equivalences between them. Izv. Ross. Akad. Nauk Ser. Mat., 66(3):131-158, 2002. translation in Izv. Math., 66(3):569-594, 2002.

[32] D. O. Orlov. Derived categories of coherent sheaves and equivalences between them. Uspekhi Mat. Nauk, 58(3(351)):89-172, 2003. translation in Russian Math. Surv., 58(3):511-591, 2003.

[33] S. Parimala and R. Sridharan. Projective modules over quaternion algebras. J. Pure Appl. Algebra, 9(2):181-193, 1976/77.

[34] C. Pedrini and C. Weibel. Invariants of real curves. Rend. Sem. Mat. Univ. Politec. Torino, 49(2):139-173 (1993), 1991.

[35] Alexander Polishchuk and Eric Zaslow. Categorical mirror symmetry: the elliptic curve. Adv. Theor. Math. Phys., 2(2):443-470, 1998.

[36] Daniel Quillen. Higher algebraic K-theory. I. In Algebraic K-theory, I: Higher K-theories (Proc. Conf., Battelle Memorial Inst., Seattle, Wash., 1972), pages 85-147. Lecture Notes in Math., Vol. 341. Springer, Berlin, 1973.

[37] Jeremy Rickard. Morita theory for derived categories. J. London Math. Soc. (2), 39(3):436456, 1989.

[38] Jonathan Rosenberg. $C^{*}$-algebras, positive scalar curvature, and the Novikov conjecture. III. Topology, 25(3):319-336, 1986.

[39] Jonathan Rosenberg. Algebraic K-theory and its applications, volume 147 of Graduate Texts in Mathematics. Springer-Verlag, New York, 1994.

[40] Jonathan Rosenberg. Real Baum-Connes assembly and T-duality for torus orientifolds. $J$. Geom. Phys., 89:24-31, 2015.

[41] Thomas Schick. Real versus complex $K$-theory using Kasparov's bivariant $K K$-theory. $A l$ gebr. Geom. Topol., 4:333-346, 2004.

[42] Andrei A. Suslin. On the K-theory of local fields. J. Pure Appl. Algebra, 34(2-3):301-318, 1984. Proceedings of the Luminy conference on algebraic $K$-theory (Luminy, 1983).

[43] Richard G. Swan. K-theory of quadric hypersurfaces. Ann. of Math. (2), 122(1):113-153, 1985.

[44] George Szeto. On generalized quaternion algebras. Internat. J. Math. Math. Sci., 3(2):237245, 1980.

[45] Charles A. Weibel. The K-Book: An introduction to algebraic K-theory, volume 145 of Graduate Studies in Mathematics. American Mathematical Society, Providence, RI, 2013.

[46] Ernst Witt. Zerlegung reeller algebraischer Funktionen in Quadrate. Schiefkörper über reellem Funktionenkörper. J. Reine Angew. Math., 171:4-11, 1934.

[47] Edward Witten. D-branes and K-theory. J. High Energy Phys., 1998(12):Paper 19, 1998.

[48] Edward Witten. Toroidal compactification without vector structure. J. High Energy Phys., 1998(2):Paper 6, 1998.

[49] Dongyuan Yao. A note on the $K$-theory of twisted projective lines and twisted Laurent polynomial rings. J. Algebra, 173(2):424-435, 1995.

Department of Mathematics, University of Maryland, College Park, MD 207424015, USA

E-mail address, Jonathan Rosenberg: jmr@math.umd.edu 